Neurol Med Chir (Tokyo) 50, 390 392, 2010

\title{
Rapid Spontaneous Resolution of Neurological Signs and Cerebral Herniation Caused by Intracerebral Hemorrhage -Case Report-
}

\author{
Hitoshi Yamahata, Masashi HiRABARU, and Kazunori ARITA* \\ Department of Neurosurgery, Hokusatsu Hospital, Isa, Kagoshima; \\ *Department of Neurosurgery, Faculty of Medicine, Kagoshima University, Kagoshima
}

\begin{abstract}
A 47-year-old man presented with sudden consciousness disturbance and left hemiplegia caused by intracerebral hemorrhage. Initial computed tomography (CT) showed a massive subcortical right temporoparietal lobe hematoma and signs of impending uncal herniation. However, he became alert and his hemiplegia improved considerably in the course of 2 hours, so conservative treatment was preferred over surgical intervention. Follow-up CT acquired on the next day revealed dramatic diminution of the intracerebral hemorrhage. Small intracerebral hemorrhages occasionally produce transient symptoms with or without disappearance of the hematoma, but the present patient had a large hematoma that decreased in size within a short time with rapid improvement of the symptoms attributed to the hematoma. The mechanism underlying the diminution of the hematoma may be related to redistribution by cerebrospinal fluid flow.
\end{abstract}

Key words: intracerebral hemorrhage, spontaneous resolution, cerebral herniation, conservative therapy, cerebrospinal fluid

\section{Introduction}

Intracerebral hemorrhage is the second most common form of stroke, and accounts for $10-20 \%$ of all events. ${ }^{1)}$ The outcome is generally prolonged or permanent focal neurological dysfunction, with neurological deficits evolving over the course of minutes or hours, and persisting for days or weeks before major improvement.9) Although patients have achieved rapid recovery after intracerebral hemorrhage, rapid spontaneous resolution of impending herniation caused by massive cerebral hemorrhage is ex-

Received July 23, 2009; Accepted October 9, 2009 tremely rare. We treated a patient with intracerebral hemorrhage whose neurological signs improved rapidly and whose hematoma decreased quickly in size.

\section{Case Report}

A 47-year-old man with alcoholic cirrhosis suddenly developed consciousness disturbance and left hemiplegia. He was transferred to our hospital one hour later. On admission he was drowsy with left hemiplegia, and Glasgow Coma Scale (GCS) score was 13 (E-3, V-4, M-6). Coagulation tests revealed prolongation of prothrombin time (13.9 sec), but normal platelet count. Liver function tests dis- 


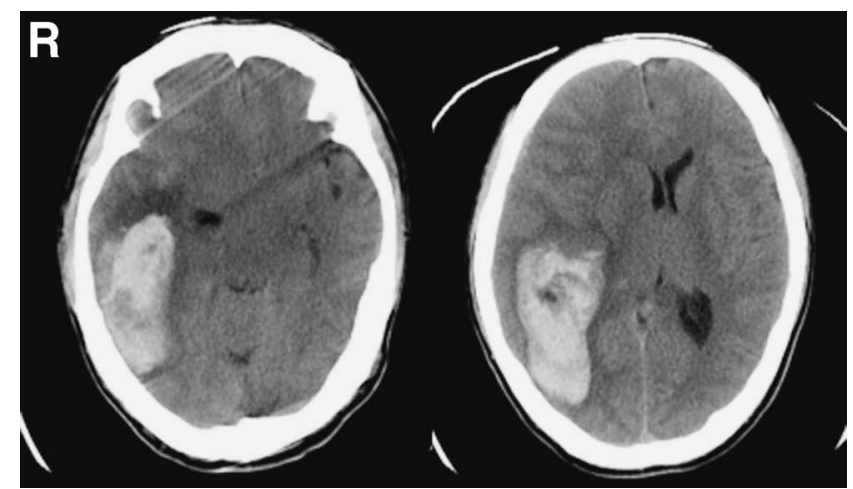

Fig. 1 Brain computed tomography scans taken on admission showing massive intracerebral hemorrhage on the right with midline shift and disappearance of the peri-mesencephalic cistern.

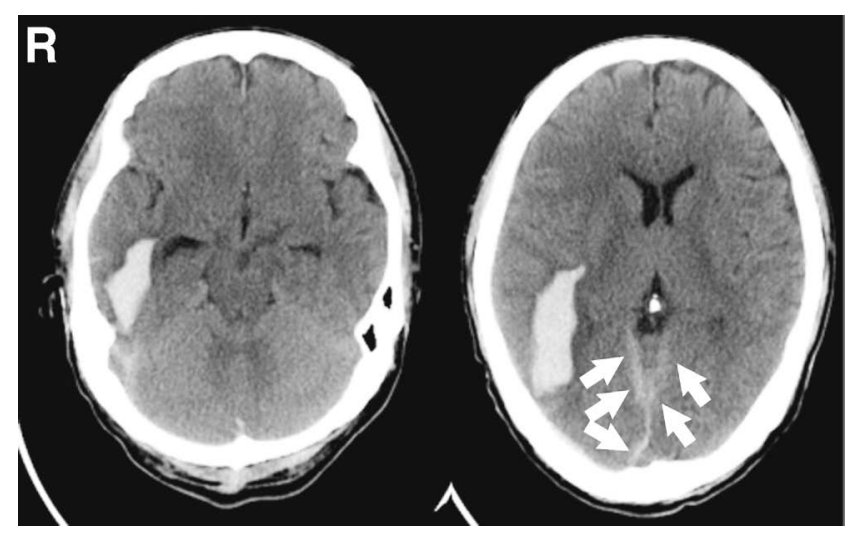

Fig. 2 Brain computed tomography scans acquired on the first day after admission showing a significant decrease in the size of the intracerebral hemorrhage and associated mass effect, and thin subdural hematoma adjacent to the cerebellar tentorium (arrows).

closed total bilirubin of $1.6 \mathrm{mg} / \mathrm{dl}$, direct bilirubin of 0.7 $\mathrm{mg} / \mathrm{dl}$, aspartate aminotransferase $21 \mathrm{IU} / \mathrm{l}$, alanine aminotransferase $81 \mathrm{U} / \mathrm{l}$, alkaline phosphatase $218 \mathrm{IU} / \mathrm{l}$, and $\partial$-glutamyl transpeptidase $13 \mathrm{IU} / \mathrm{l}$. Computed tomography (CT) revealed a massive hematoma in the right temporoparietal lobe with severe midline shift and disappearance of the peri-mesencephalic cistern (Fig. 1). Magnetic resonance angiography showed stenosis of the left middle cerebral artery, but no aneurysms or arteriovenous malformations were detected on the affected side. The diagnosis was subcortical hemorrhage unrelated to obvious vascular abnormalities.

Emergent surgery was planned to relieve the transtentorial herniation, despite the prolonged prothrombin time. However, his symptoms dramatically improved one hour after admission and his GCS score rose to 15 (E-4, V-5, M-6). He could move his left arm and leg in response to instructions. We chose to proceed with conservative therapy under strict blood pressure control because his symptoms had improved, also because the status of his liver function was not fully known, so the safety of craniotomy under general anesthesia was uncertain. On the next day, his symptoms remained stable and follow-up CT revealed a remarkable decrease in the size of the hematoma and reappearance of the peri-mesencephalic cistern (Fig. 2). He complained of occasional headache that did not respond to medication over the next few days, but his overall clinical course was uneventful. He was enrolled in a rehabilitation program and was discharged after 3 weeks with slight left hemiparesis.

\section{Discussion}

The present patient with intracerebral hemorrhage showed very rapid improvement without interventional therapy. CT on admission revealed a large subcortical hemorrhage and signs of impending uncal herniation. Unexpectedly, CT acquired the next day disclosed remarkable diminution of the hematoma.

Intracerebral hemorrhage is a known cause of transient focal neurological attacks. ${ }^{5,10}$ ) Nine patients with intracerebral hemorrhage recovered rapidly ${ }^{9}$ and 2 patients with intracerebral hemorrhage had focal neurological symptoms and signs which resolved spontaneously within 24 hours. ${ }^{5)}$ However, these patients had relatively small hematomas, whereas our patient had a large hematoma associated with impending transtentorial herniation.

The true incidence of rapid resolution of intracerebral hemorrhage remains unknown, but hemorrhages are known to diminish spontaneously, if only rarely. ${ }^{3,4}$ A patient with petrous bone fracture after head trauma showed spontaneous cure of intracerebral hematoma by drainage into the middle ear. ${ }^{3)}$ However, in our case, the intracerebral hemorrhage was not related to head trauma, so appears to be a large hematoma which decreased spontaneously resulting in rapid disappearance of symptoms.

Several mechanisms have been proposed to explain the rapid spontaneous resolution and redistribution of subdural hematoma..$^{2,6-8,11)}$ For example, hematoma resolution has been attributed to intracranial redistribution and washing out by cerebrospinal fluid (CSF) through a torn arachnoid membrane. ${ }^{2,6-8,11)}$ Although this mechanism has not been proposed, the same pathophysiology may underlie the rapid resolution of intracerebral hemorrhage. Our patient presented with a subcortical hematoma with a margin very close to the subarachnoid space. We propose that pressure from the massive hematoma caused rupture of the adjacent cortical surface and pia mater, resulting in migration and dispersion of the main part of the hematoma into the subarachnoid and subdural spaces. The appearance of a thin subdural hematoma adjacent to the cerebellar tentorium on the second CT scan (Fig. 2) may support this hypothesis.

Co-mingling of hematoma with the CSF is key in the redistribution process. ${ }^{2,6-8,11)}$ CSF liquidizes the hematoma and the blood clots are redistributed to the subarachnoid and subdural spaces. ${ }^{7)}$ In addition, our patient had a history of liver cirrhosis, so coagulopathy may have enhanced the mobility of the hematoma. Rapid resolution of an acute subdural hematoma has never been attributed to the 
delayed coagulation cascade, but patients with liver cirrhosis or chronic alcoholism occasionally manifest an association with liver function disorders. ${ }^{8,11)}$

The present case suggests that clinicians should be aware that large intracerebral hemorrhages may diminish spontaneously, although rare and of unclear pathophysiology. Factors underlying spontaneous improvement in patients with life-threatening cerebral hemorrhage must be identified before performing emergency surgery. Close monitoring and conservative management may be advisable in patients whose clinical status improves spontaneously.

\section{Acknowledgments}

We are grateful to Ms. U. Petralia for editorial assistance.

\section{References}

1) Asdaghi N, Manawadu D, Buther K: Therapeutic management of acute intravertebral haemorrhage. Expert Opin Pharmacother 8: 3097-3116, 2007

2) Berker M, Gulsen S, Ozcan OE: Ultra-rapid spontaneous resolution of acute posttraumatic subdural hematomas in patient with temporal linear fracture. Acta Neurochir (Wien) 145: 715-717, 2003

3) Bose B, Kraut W, Osterholm JL: Intracerebral hematoma: Spontaneous cure by drainage into the middle ear. Neurosurgery 10: 103-104, 1982
4) Dennis MS, Bamford JM, Molyneux AJ, Warlow CP: Rapid resolution of signs of primary intracerebral haemorrhage in computed tomograms of the brain. Br Med J 295: 379-381, 1987

5) Gunatilake SB: Rapid resolution of symptoms and signs of intracerebral haemorrhage: Case reports. Br Med J 316: 1495-1496, 1998

6) Imai K: Rapid spontaneous resolution of signs of intracranial herniation due to subdural hematoma. Neurol Med Chir (Tokyo) 43: 125-129, 2003

7) Kato N, Tsunoda T, Matsumura K, Yanaka K, Nose T: Rapid spontaneous resolution of acute subdural hematoma occurs by redistribution. Neurol Med Chir (Tokyo) 41: 140-143, 2001

8) Niikawa $S$, Sugimoto $S$, Hattori $T$, Ohkuma A, Kimura $T$, Shinoda J, Funakoshi T: Rapid resolution of acute subdural hematoma. Neurol Med Chir (Tokyo) 29: 820-824, 1989

9) Scott WR, Miller BR: Intracerebral hemorrhage with rapid recovery. Arch Neurol 42: 133-136, 1985

10) Sohn YH, Kim SM, Kim JS, Kim DI: Benign brainstem hemorrhage simulating transient ischemic attack. Yonsei Med J 32: 91-93, 1991

11) Tsui EYK, Ma KF, Cheung YK, Chan JHM, Yuen MK: Rapid spontaneous resolution and redistribution of acute subdural hematoma in a patient with chronic alcoholism: A case report. Eur J Radiol 36: 53-57, 2000

Address reprint requests to: Hitoshi Yamahata, M.D., Division of Neurosurgery, Kagoshima City Hospital, 20-17 Kajiya-cho, Kagoshima, Kagoshima 892-8580, Japan. e-mail: yamahata-nsu@umin.net 